\title{
Adjustability of a discrete particle swarm optimization for the dynamic TSP
}

\author{
Lukasz Strąk $^{1}$ - Rafał Skinderowicz ${ }^{1}$ - Urszula Boryczka ${ }^{1}$
}

Published online: 28 July 2017

(C) The Author(s) 2017. This article is an open access publication

\begin{abstract}
This paper presents a detailed study of the discrete particle swarm optimization algorithm (DPSO) applied to solve the dynamic traveling salesman problem which has many practical applications in planning, logistics and chip manufacturing. The dynamic version is especially important in practical applications in which new circumstances, e.g., a traffic jam or a machine failure, could force changes to the problem specification. The DPSO algorithm was enriched with a pheromone memory which is used to guide the search process similarly to the ant colony optimization algorithm. The paper extends our previous work on the DPSO algorithm in various ways. Firstly, the performance of the algorithm is thoroughly tested on a set of newly generated DTSP instances which differ in the number and the size of the changes. Secondly, the impact of the pheromone memory on the convergence of the DPSO is investigated and compared with the version without a pheromone memory. Moreover, the results are compared with two ant colony optimization algorithms, namely the $\mathcal{M} \mathcal{A} \mathcal{X}-\mathcal{M I N}$ ant system (MMAS) and the population-based ant colony optimization (PACO). The results show that the DPSO is able to find high-quality solutions to the DTSP and its performance is competitive with the performance of the MMAS and the PACO algorithms. Moreover, the pheromone memory has a positive impact on
\end{abstract}

Communicated by V. Loia.

Łukasz Strạk

lukasz.strak@us.edu.pl

Rafał Skinderowicz

rafal.skinderowicz@us.edu.pl

Urszula Boryczka

urszula.boryczka@us.edu.pl

1 Institute of Computer Science, University of Silesia in Katowice, Bȩdzińska 39, 41-205 Sosnowiec, Poland the convergence of the algorithm, especially in the face of dynamic changes to the problem's definition.

Keywords Discrete particle swarm optimization . Pheromone memory - Dynamic traveling salesman problem . Population-based ant colony optimization

\section{Introduction}

A problem in which input data are variable (i.e., time dependent) is called a dynamic optimization problem (DOP). The aim of optimization in the DOP is to continuously track and adapt to changes and quickly find the best solution ( $\mathrm{Li} 2011)$. DOPs can be divided into two groups (Mori and Kita 2000): online (direct adaptation) and offline (indirect adaptation). In the first case, the changes are hard to predict and could happen at any moment during the algorithm runtime. In the second case, the changes happen at specified intervals between which the problem definition does not change. Many other DOPs classification criteria were described by Yang and Yao (2013), including

- time linkage - is the change affected by the current solution;

- cyclicity - is the change to the problem cyclic or not;

- factors - do the changes affect the number or values of variables, constraints, etc.

DOPs were investigated mostly in the context of the continuous optimization. In this work, we focus on the discrete DOP that is the DTSP in the offline version.

Computational intelligence methods are a set of techniques that are used to solve optimization problems. They include algorithms that were inspired by the collective behav- 
ior of animals which are able to solve complex problems in their natural environment by cooperating with one another. It is often beyond the capabilities of a single individual to solve these problems, i.e., to accomplish tasks such as foraging and nest building, yet this is not problematic for a group of animals. When a combination of relatively simple behaviors of particular individuals produces complex interactions, then we can talk about swarm or collective intelligence.

The PSO is a good example of a computational intelligence algorithm inspired by the natural behavior of animal herds, e.g., a school of fish or a flock of birds. Both fish and birds are able to coordinate the movement of the flock without the need to communicate directly. The PSO was proposed by Kennedy and Eberhart (1995) in order to solve the function optimization problem. In the PSO, a swarm (population) of particles moves around in the solution search space. Each particle has a position that corresponds to a solution to the problem being solved and velocity that shows the direction and velocity of the movement. A movement of a particle is also influenced by its local best known position, typically denoted by $p$ Best, and the best position found by the swarm (usually denoted by $g$ Best). This indirect interaction of the particles is intended to guide the swarm toward solutions of good quality. The relative simplicity and efficiency of the PSO was a key to its many successful applications, also in solving the discrete combinatorial optimization problems.

The behavior of certain species of ants that forage for food provided inspiration for creating ant colony algorithms (Dorigo and Stützle 2010). While searching for food, these ants lay a certain amount of pheromone as they move. At the beginning, they randomly choose the direction of the search process. With each subsequent trip from the nest to a food source and back, over time ants will deposit the largest amount of pheromone on the shortest path because much of the pheromone that has been laid on longer paths will evaporate before being reinforced by returning ants. After a while, a given pheromone trail will be reinforced to such an extent that when beginning its trip, each subsequent ant will follow that pheromone trail that has been laid down on the shortest path from the nest to a food source. In this case, pheromone acts as a means of indirect communication and in a way as collective memory. Ant colony algorithms and particle swarm optimization are the most popular methods that were inspired by natural collective animals behavior.

It is easy to compare two algorithms based on computational experiments if these algorithms can be run on the same input data. As for the traveling salesman problem, the TSPLIB library facilitates such comparisons (Reinelt 1995). Apart from data themselves, this library also provides optimal solutions for most problems. However, there is no such library for the dynamic traveling salesman problem. One of the aims of this paper is to create a new approach for testing the quality of results that are obtained for the DTSP.
A library of the DTSP instances that have been generated contains information about optimal solutions for every subproblem which allows for a simple and precise evaluation of the performance of the algorithms. This library was used to compare the swarm intelligence algorithms investigated in this paper, i.e., the DPSO, the MMAS and the PACO.

\subsection{Contributions}

Compared to our previous articles on the DPSO for the DTSP (described briefly in Sect. 2), the research has been extended in a few directions. Firstly, we closely compare the convergence and efficiency of the two DPSO versions: the DPSOR-, in which the pheromone values are preserved between the subsequent modifications of the input data (problem) changes, and the DPSOR+, in which the pheromone is reset after each change. Secondly, we introduce a systematic way of generating new DTSP instances (a DTSP instances generator), which allows to precisely control the number and range of the changes. Thirdly, based on the newly generated DTSP instances, we investigate how the performance of the DPSOR+ and DPSOR - changes depending on the number of dynamic changes to the problem being solved. Lastly, based on statistical analysis, we compare the performance of the DPSO algorithms with the MMAS and PACO algorithms that were proven to perform competitively when solving many static and dynamic combinatorial optimization problems, including the TSP and DTSP.

The structure of this paper is as follows: The second section presents a review of literature on the dynamic traveling salesman problem and discrete particle swarm optimization algorithm. The third section describes the DTSP, whereas the fourth section contains a description of the tested algorithms that solve the DTSP. The tests that were carried out are presented in the fifth section, and the final section presents a summary and conclusions.

\section{Related work}

The dynamic traveling salesman problem was first described by Psaraftis (1988). This problem is based on a change in both the number of vertices and a distance matrix that occurs over time. Each change can modify the optimal route, and the knowledge of the optimum is useful to be able to monitor the quality of the results that are being produced by an algorithm. Younes et al. (2003) proposed a procedure in which the optimal route does not have to be known for each subproblem. A modification is carried out for half the number of subproblems (adding, removing or changing a given distance), and each of these changes is undone in the second half of the procedure. Data are the same in the first and the last subproblem. The algorithm is run for each subprob- 
lem, but distances are only compared against the optimum for the last subproblem. Another approach combines static data with data obtained from satellites orbiting the Earth. Satellite orbits are given by a formula and the optimal route only changes within these orbits (Kang et al. 2004). Unfortunately, the dynamics of this problem is low; for example, the CHN146 +3 problem contains 146 cities and 3 variable points. Yet another approach involves estimating the optimal value. In order to do this, one can use the Held-Karp algorithm that is based on the concept of 1-tree. This approach was adapted in Boryczka and Strak (2013). An advantage of taking this approach is that one can quickly estimate the optimal value, whereas its greatest drawback is that there is no approximation error margin. However, an improved method which was proposed by Helsgaun (2000) allows one to estimate the optimum length with an error of less than $1 \%$, but cannot predict what the results will be for other instances. Another method was proposed by Guntsch et al. (2001) when solving the DTSP with the ACO. The approach involves simulating dynamic changes to the problem by exchanging a number of cities between the current problem instance and a spare pool of cities. As the method affects the optimum, Guntsch et al. (2001) evaluated their algorithms based on the relative differences in the length of the successive solutions. A more general method for generating dynamic versions of combinatorial optimization problems (COPs), including the TSP, was proposed by Younes et al. (2005). The basic idea, in the context of genetic algorithms, exploits the fact that most optimization methods involve some form of mapping from the problem solution space to the individuals used in the algorithm, e.g., a permutation of nodes. To simulate dynamic changes to the problem, the mapping function is modified by exchanging labels (indices) of some nodes; thus, the individuals represent different solutions, but the fitness landscape, and so the optimum, of the problem instance does not change. Mavrovouniotis et al. (2012) proposed a more flexible approach in which the encoding of the problem instance is modified, instead of the encoding of the individuals. In the case of the TSP and VRP, it involves swapping the locations of pairs of nodes. The method keeps the optimum intact and allows for precise control over the number of dynamic changes to the problem instance. However, this approach does not necessarily reflect each real-world scenario.

The PSO algorithm has been adapted several times to solve the TSP. The first version of the DPSO was proposed by $\mathrm{Hu}$ et al. (2004). All particles were coded as binary strings. The predefined velocity was interpreted as the probability of a bit state transition from zero to one and from one to zero, but a sigmoid function could be used to restrict the values to 0 and 1. Zhong et al. (1997) proposed a new algorithm for the TSP in which the position of the particle was not a permutation of numbers but a set of edges. It used the parameter $c_{3}$, named by the authors a mutation factor, which allowed control the balance between the exploration and the exploration in a discrete search space. Descriptions of the most PSO algorithms for solving the TSP can be found, together with the results, in Goldbarg et al. (2008). A hybrid of the PSO algorithm and pheromone was used in Kalivarapu et al. (2009). This approach was implemented to deal with the problem of optimizing a function in a continuous space. The survey of the PSO approaches to the dynamic continuous optimization can be found in Blackwell et al. (2008).

There are relatively few applications of the DPSO in the realm of dynamic COPs. Okulewicz and Mańdziuk (2013) proposed a two-phase PSO to solve the dynamic VRP. In the first phase, the PSO was responsible for the assignment of the customers to vehicles, while in the second phase separate instances of the PSO were used to find the order in which the customers should have to be visited (static TSP). A similar approach was proposed in Demirtaş et al. (2015). Khouadjia et al. (2010) proposed an adaptive PSO for solving the VRP with dynamic requests. The algorithm stored previous solutions in the form of a memory continuously updated during the algorithm runtime. The old solutions in the memory were used as starting points when a change to the problem definition was detected, as the changes could result in a new optimum being in the vicinity of the old one. A recent thorough survey on swarm intelligence methods for solving the dynamic COPs (continuous and discrete) can be found in Mavrovouniotis et al. (2017).

The literature on the DPSO algorithm for the DTSP is very limited. This paper constitutes an extension of our earlier work on the DPSO algorithm. The initial version of the DPSO algorithm was presented in Boryczka and Strạk (2012) and Boryczka and Strạk (2013). That version differed from the one presented in this article in the pheromone update formula and the solution construction process. In Boryczka and Strak (2015a), a new version of the DPSO algorithm was presented, in which the computations were reset based on the generated solutions entropy. The most recent work of Boryczka and Strak (2015b) concerned the problem of the automatic algorithm parameter values adaptation. In this article, we extend the previous work on the DTSP algorithm, as presented in Boryczka and Strak (2015a, b), in several ways.

Various solutions were proposed to adapt the ant colony algorithms, including the ACO and PACO, to solving the DTSP. One of the simplest ways of dealing with dynamic changes is to reset the pheromone memory after a change was detected; unfortunately, this strategy is not very effective because it leads to the loss of all the information about the previous version of the problem that has been collected in the pheromone memory. Guntsch et al. compared a strategy that involves resetting the entire pheromone memory with two strategies of modifying the pheromone only in the neighborhood of a point where a change in the problem's configuration has been detected; this modification involves adding or 
removing a TSP node (Guntsch and Middendorf 2001). However, strategies that involved resetting only a selected part of the pheromone matrix did not produce significantly better results than those obtained in the case of resetting the entire memory. The authors of the PACO algorithm tested its usefulness in solving the DTSP and QAP (Guntsch and Middendorf 2002). Another idea proposed in the literature was to diversify the ants population (Eyckelhof et al. 2002; Mavrovouniotis and Yang 2010; Boryczka and Strąk 2015a). After each change to the problem's definition, the diversity of the ants population was increased to allow for a faster adaptation to the new version of the problem. In the recent years, the ACO algorithms were successfully applied to other dynamic combinatorial optimization problems. A good example is the dynamic generalized traveling salesman problem (DGTSP) for which Pintea et al. (2007) proposed an algorithm based on the ant colony system (ACS). The computational experiments on a set of the DGTSP instances, created based on the TSPLIB repository, confirmed efficiency of the suggested algorithm. A similar approach was also confirmed to be useful when solving the generalized vehicle routing problem (Pop et al. 2009). Time is a scarce resource when solving the dynamic combinatorial optimization problems; thus, parallel computations are very useful to accelerate the process of finding solutions to a new version of a problem, i.e., after a dynamic change. A parallel ACO was proposed in Pintea et al. (2012) in order to solve the Euclidean DTSP instances with a few thousand nodes. A recent survey of the parallel ACO algorithms can be found in Pedemonte et al. (2011).

\section{Dynamic TSP}

The classical TSP problem is typically modeled using a complete, weighted graph $G$ defined as follows:

$G=\langle V, E, w\rangle$,

where:

$V$ is a set of vertices representing cities,

$V \times V \subset E$ is a set of edges representing roads between cities; $|E|=\left(\begin{array}{l}n \\ 2\end{array}\right), n=|V|$ (a complete graph),

$w$ is a function of weights: $E \rightarrow \mathbb{R}, \quad \forall e \in E, w(e)$ is the weight of an edge.

The problem involves finding the minimum Hamiltonian cycle in graph $G$, i.e., the shortest closed path that goes through all vertices exactly once. The problem that is analyzed in this paper is symmetric: $d_{a b}=d_{b a}$ (where $d_{i j}$ denotes the distance between cities $i, j$ ) and Euclideanany set of three cities, $\{a, b, c\} \subseteq V$, satisfies the triangle inequality: $d_{a c} \leq d_{a b}+d_{b c}$.
The DTSP formulation is an answer to many real-world scenarios, in which the external conditions alter the original definition of the static TSP. For example, the travel time between a pair of nodes may increase due to a higher traffic or a car accident. In another scenario, new customers (nodes) have to be accounted for or some of the current ones become unavailable. In our work, we assume that the changes to the problem definition are not very frequent.

The model of the DTSP consists of a sequence of modified static TSP subproblems, and every change in these subproblems means a modification of the distances and/or the number of vertices (Psaraftis 1988). In formal terms, this problem can be defined in the following way ( $\mathrm{Li}$ et al. 2006):

$D(t)=\left\{d_{i j}(t)\right\}_{n(t) \times n(t)}$,

where:

$t$ denotes the parameter of time or the subproblem number,

$i, j$ denote the ends of an edge,

$n(t)$ denotes the number of vertices as a function of time.

A change is made randomly and only with regard to a selected subset of vertices. Only the variant that involves a change in a distance matrix is analyzed in this paper.

\subsection{DTSP Instances Generator}

In the context of the DTSP, different algorithms are usually compared in such a way that the coordinates of vertices (cities) are randomly modified for each execution of an algorithm; therefore, each time, the search space changes in a slightly different manner. The tests that were presented in Bilu and Linial (2012) show that each change in the configuration of the problem entails a change in the location of the global optimum which is difficult to predict. It also has other consequences, such as a change in the problem's difficulty level. The difficulty of the TSP can be analyzed in the context of, for example, the distances between local minima as well as between local minima and the global optimum. If the distance between the global optimum and the other optima is large, the search process can easily get stuck in a local optimum (Bilu and Linial 2012). Thus, the problem's difficulty increases in a way that is hard to predict. In order to facilitate a comparison between the analyzed algorithms in terms of efficiency in the context of the DTSP, the following strategy was adapted. A set of TSP instances was selected from the TSPLIB library (Reinelt 1995), on the basis of which instances of the DTSP were created (the TSPLIB format was maintained) by randomly changing the location of a predetermined number of vertices in accordance with Algorithm 1. The resulting DTSP instance consisted of 11 
Fig. 1 On the left-hand side is a visualization of the optimal route for the problem (ch130), whereas on the right-hand side, the edges that differentiate the optimal solution that had been created before the changes were made from the one that was created after those changes are marked in red (color figure online)

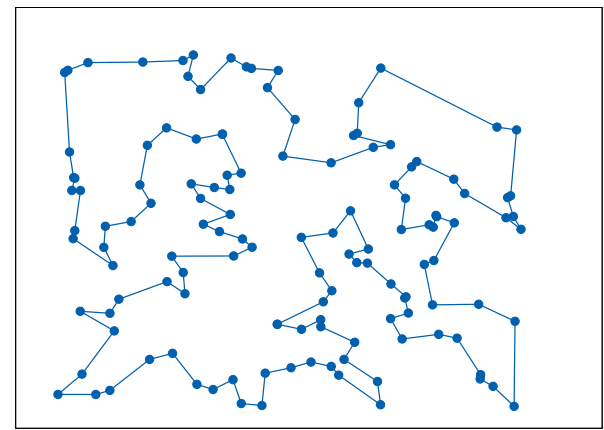

consecutive subproblems; the first subproblem was identical to the TSP instance from the TSPLIB library, whereas each subsequent subproblem was created by changing the location of a predetermined proportion of vertices. New values of coordinates $\left(p_{x}^{\prime}, p_{y}^{\prime}\right)$ of a given vertex (city) were calculated in accordance with the formula:

$$
\begin{aligned}
r & =f_{\text {inf }} \cdot \operatorname{rand}\left(0, d_{\text {avg }}\right) \\
\phi & =\operatorname{rand}(0,2 \pi) \\
p_{x}^{\prime} & =p_{x}+r \cdot \cos (\phi) \\
p_{y}^{\prime} & =p_{y}+r \cdot \sin (\phi),
\end{aligned}
$$

where $\left(p_{x}, p_{y}\right)$ denote the previous location of that vertex, $d_{\text {avg }}$ represents the average distance between all points and $\phi$ stands for the angle at which the point will be shifted. Parameter $f_{\text {inf }}$ is a scaling parameter that controls the extent of changes; in this paper, a value of 0.3 was adapted.

Compared to some of the more advanced methods mentioned in Sect. 2, the location changes in the proposed generator influence the optima of the subproblems. Although it is clearly a disadvantage compared to the optima-preserving methods, it may be closer to many real-world scenarios in which weights of the TSP edges are modified (Tinós et al. 2014). For each subsequent subproblem, an optimal solution was determined by using the Concorde algorithm (Applegate et al. 2006).

Equation 2 enables a precise control over the extent of changes to the DTSP definition. Moreover, it preserves the triangle inequality of the distances between the nodes; thus, the problem after a change should not be more difficult to solve than the original version. This makes it easier to focus on the behavior of the studied algorithms.

Figure 1 shows the optimal solutions for two consecutive subproblems for the berlin52 instance. The edges that differentiate the new solution from the previous one have been highlighted. The current library ${ }^{1}$ contains 64 problems which are based on eight problems from the TSPLIB library: berlin52, kroA100, ch130, kroA200, gil262, gr202, pcb442 and $g r 666$; for each of them, a total of eight DTSP instances

\footnotetext{
1 The library also contains charts presenting optimal solutions, and it can be made available upon request to the authors.
}
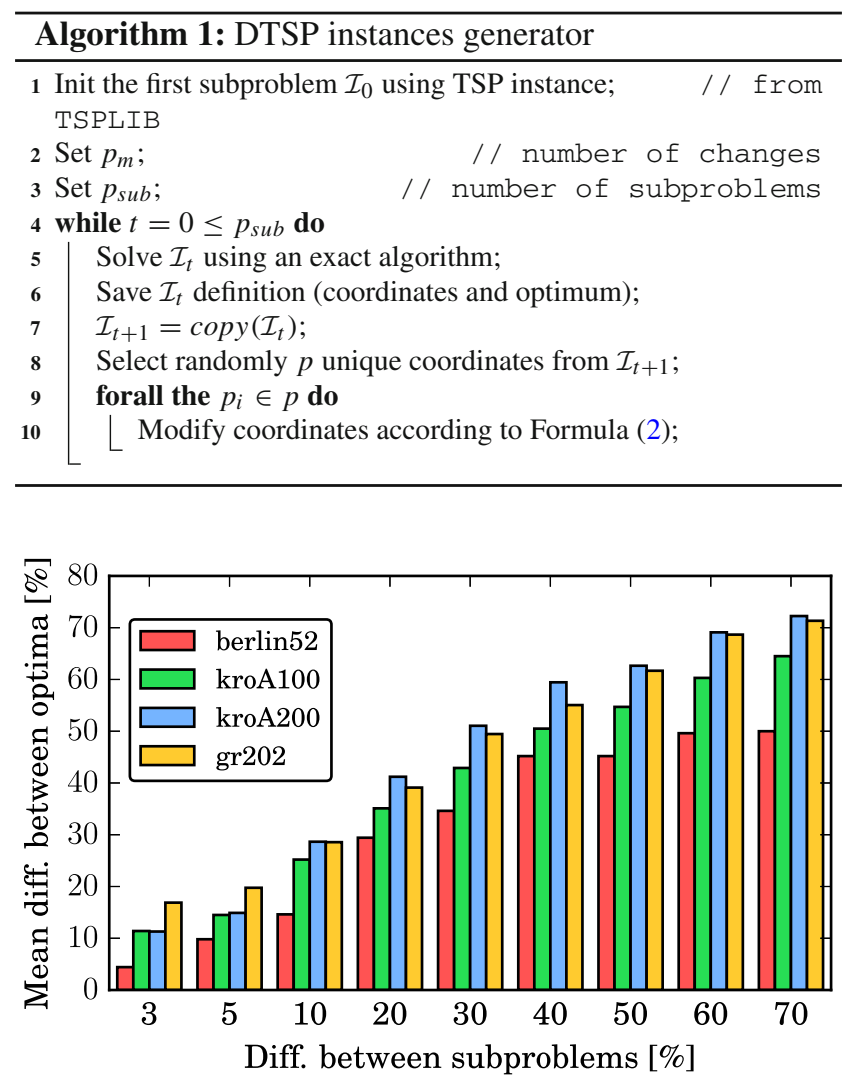

Fig. 2 Mean difference between consecutive DTSP subproblems' global optima as measured by the number of different (new) edges versus the differences between consecutive subproblems (which is equal to the percent of the total number of vertices whose locations underwent a change)

have been generated, which differ in terms of the percentage of vertices that undergo modifications. The following percentages were assumed: $3,5,10,20,30,40,50,60$ and $70 \%$. For example, for a value of $5 \%$, consecutive subproblems differ in terms of the coordinates $\lfloor 0.05 \cdot n\rfloor$ of vertices, where $n$ denotes the total number of vertices (problem size). Figure 2 shows how much the consecutive optima differ from one another in terms of the number of vertices that undergo changes. As can be seen, the greater the number of coordinates that undergo changes, the larger the differences between the consecutive optima, and these differences 
increase with the size of the problem. For example, if subsequent subproblems are created for the problem kroA200 by changing the location of $30 \%$ of vertices, then subsequent optimal solutions contain approximately $50 \%$ of new edges relative to the previous solution.

\section{Particle Swarm Optimization}

A pheromone is a characteristic component of ant colony algorithms, in which a set of ants lay virtual pheromone trails on elements of the search space (solution space). The classical PSO algorithm does not use pheromone, but there are hybrid versions of this algorithm that do. This is true for both continuous and discrete optimization algorithms.

\subsection{DPSO}

Many concepts had to be redefined in order to adapt PSO algorithm to a discrete search space. The algorithm that was proposed in Zhong et al. (1997) is based on sets of edges. A single edge $e$ is an ordered 3-tuple $(a, x, y)$, where $a \in[0,1]$ denotes the probability of choosing this edge when moving to the next position and $x, y \in V, \forall_{x, y} x \neq y$ denote the ends of that edge. In this algorithm, the solution to the TSP: $\{(1,2)$; $(2,3) ;(3,4) ;(4,1)\}$ takes the following form: $\{(1,1,2)$, $(1,2,3),(1,3,4),(1,4,1)\}$. The position of particle $X$ is represented by a set of edges that constitute a Hamiltonian cycle. Velocity $V$ represents the search direction. It is a set of edges that can contain a partial solution to the problem (it does not contain all the edges that are needed to create a Hamiltonian cycle) or redundant edges. The common feature of all particle swarm optimization algorithms is that they work based on an equation that describes a particle's movement in the search space. The original DPSO algorithm (Zhong et al. 1997) does not use pheromone which provides an adaptation mechanism for the DTSP. Formulas (3) and (4) present the authors' own discrete version of these equations that take into account pheromone (firstly described in Boryczka and Strạk (2012)).

$$
\begin{aligned}
V_{i}^{k+1} & =c_{2} \operatorname{rand}() \cdot\left(g \text { Best }-X_{i}^{k}\right) \\
& +c_{1} \operatorname{rand}() \cdot\left(\text { pBest }_{i}-X_{i}^{k}\right) \\
& +\omega \cdot V_{i}^{k} \\
X_{i}^{k+1} & =\underbrace{\Delta \tau^{k}\left(V_{i}^{k+1}\right)}_{\text {step a }} \oplus \underbrace{c_{3} \operatorname{rand}() \cdot X_{i}^{k}}_{\text {step b }}
\end{aligned}
$$

where $i$ denotes the particle number, $k$ - the iteration number and $\operatorname{rand}()-$ a random variable within the range $[0,1]$. The sets of edges pBest and $g$ Best represent particle $i$ 's best position and the best solution that has been found, the addition and subtraction operators denote the sum and difference of the sets, and coefficient $\omega$ is called the inertia weight. Parameters $c_{1}$ and $c_{2}$ are cognitive and social scaling coefficients, respectively; they assign the appropriate values to parameter $a$ for each element of the pBest and gBest sets. Operator $\oplus$ adds the missing edges to the next position; these edges are necessary for creating the correct route for the TSP. For this purpose, the operator uses the edges that were used to calculate the previous position. The way in which the operator works will be explained later in this paper. The function $\Delta \tau^{k}$ increases the probability of going to the next position by using pheromone which has already been employed in ant colony algorithms. The function is used in row 10. Algorithm 2 for calculating a particle's next velocity as well as at the stage of edge filtering. The reinforcement function is given by formula:

$$
\begin{aligned}
\Delta \tau^{k}\left(V_{i}^{k+1}\right)= & a+\left[\left(\tau_{x y}-0.5\right) \cdot \frac{k}{p_{i t}}\right], \\
& \forall(a, x, y) \in V_{i}^{k+1} \subseteq E
\end{aligned}
$$

where $(a, x, y)$ denotes an edge that belongs to the set of velocities, $\tau_{x y}$ represents the value of pheromone that has been read from the pheromone matrix, $k$ stands for the algorithm iteration number and $p_{i t}$ denotes the number of all iterations of the algorithm. Coefficient $\frac{k}{p_{i t}}$ is a scaling coefficient that controls the strength of (positive or negative) reinforcement based on the number of iterations of the algorithm that have been carried out. As a result of executing function (5), a set of edges is created with coefficient $a$ (i.e., the probability of using a given edge to go to the next position) which has been positively or negatively reinforced. The value of the reinforcement is within the range $[-0.5,0.5]$. The pheromone that has been assigned a negative value (repellent) is interpreted as a penalty function which repels those edges that do not improve the quality of the obtained solution. In this way, the algorithm only chooses those edges that have a high chance of being a part of good quality solutions as representing a given particle's next position. Uncertainty about the quality of edges is reflected in the pheromone value. The higher the value, the more probable it is that a given edge will improve the solution. In subsequent iterations of the algorithm, this value is modified as a result of evaporating and reinforcing the pheromone. Those edges that often constitute a part of the best solution have a high pheromone value, and therefore, they also receive positive pheromone reinforcement (Formula 5). The ability to self-adapt is an important characteristic of pheromone in the context of the DTSP. If in the initial iterations of the algorithm a certain edge is often a part of the best solution, then it will be assigned a high value in the pheromone matrix. If, however, that edge ceases to be a part of the best solution at some point, its value in the pheromone matrix will begin to decrease, until it finally 


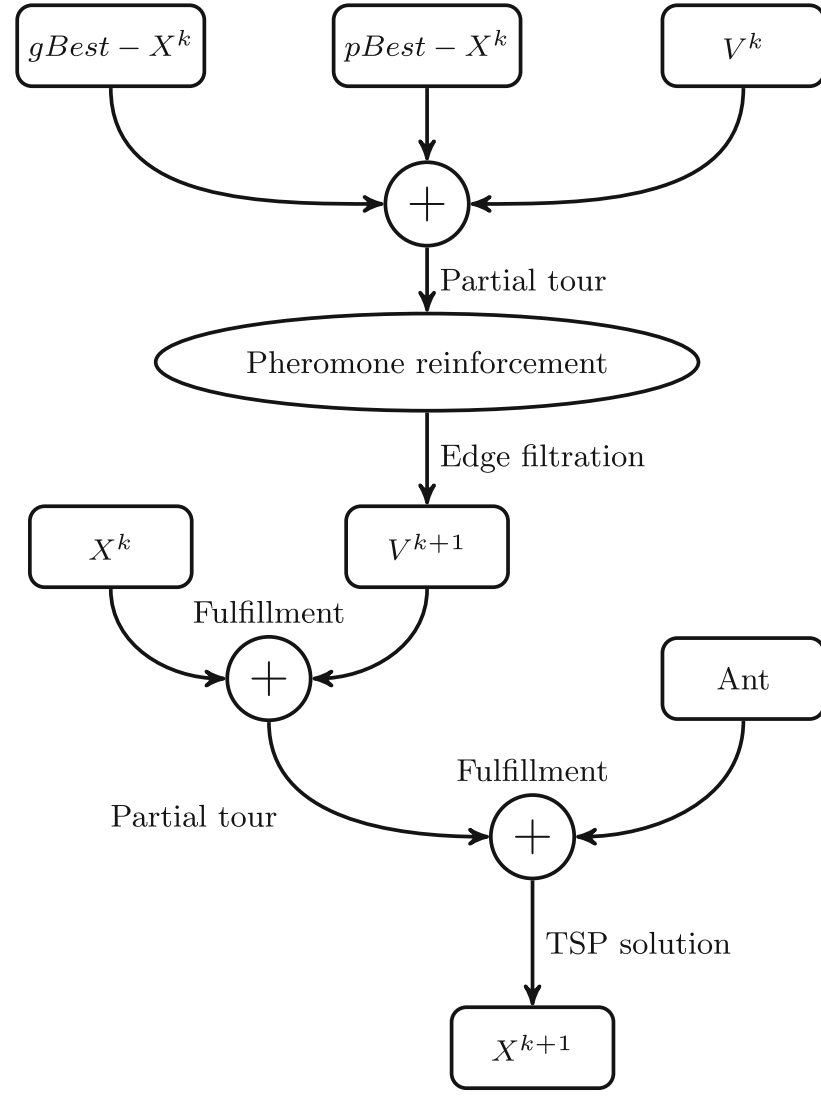

Fig. 3 Calculation of a new position in the DPSO algorithm. This operation is carried out for all particles in the swarm. All variables are sets

reaches the minimum value $\tau_{\min }$. Figure 3 shows a graphical representation of the DPSO algorithm.

The process of updating the pheromone matrix is as follows: In the first iteration of the algorithm, each element of the matrix is assigned a default value, i.e., $\tau_{\max }$, which is also the initial value. After each subsequent iteration, the pheromone is evaporated by multiplying the pheromone matrix by coefficient $\rho<1$, and then, the edges that are a part of the best solution that has been found are reinforced. The values that are stored in the matrix are within the range $\left[\tau_{\min }, \tau_{\max }\right]$. The pheromone updating process is identical to the working of the $\mathcal{M A X}-\mathcal{M I N}$ Ant System algorithm which was developed by Stützle and Hoos (2000).

Algorithm 2 shows how the DPSO algorithm uses formulas (3) and (4). The first steps involve creating a random particle swarm, based on which the best position is selected, i.e., $g$ Best. Then, the next velocity of each particle is calculated in accordance with formula (3) (line 5. Algorithm 2). The operation of multiplying a given number by a set is carried out as a multiplication of that number by each coefficient $a$ for elements of that set (edges). The probability of choosing a given edge for a particle to go to the next position depends on coefficients $c_{1}, c_{2}, c_{3}$ and a random variable, i.e., $\operatorname{rand}()$.

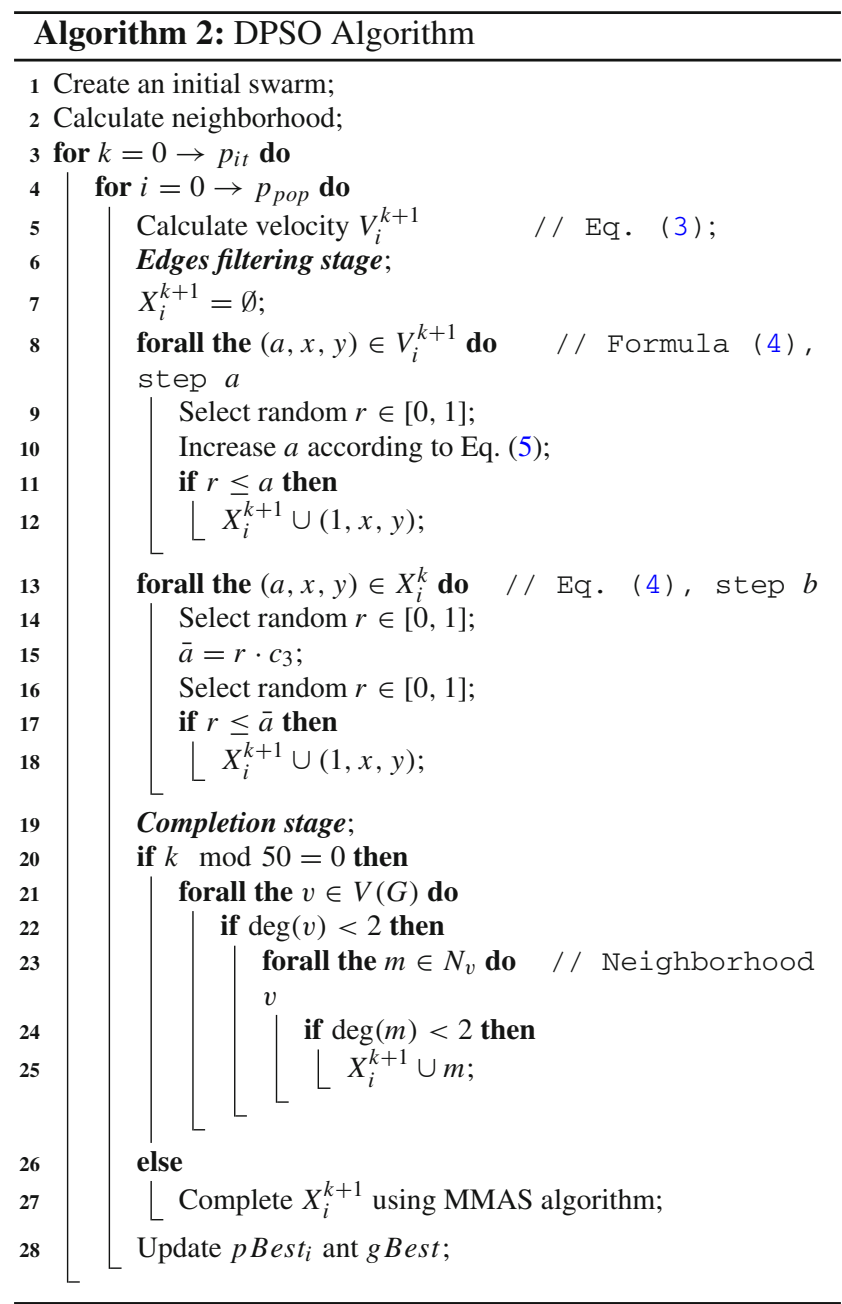

This process is responsible for a random selection of edges. In this way, a particle's next velocity is computed, based on which its next position is established. A new solution is created in two stages, i.e., filtering and completion. At the first stage, each edge belonging to the set of velocities is copied to a particle's next position if the value of coefficient $a$ for that edge is higher than the value of random variable $r$ (rows 11. and 17.).

The filtering stage is followed by the completion stage, which is aimed at adding the missing edges so as to create a complete Hamiltonian cycle. The algorithm that was proposed by Zhong et al. (1997) uses the nearest neighbor heuristic for this purpose. The solution that is proposed in this paper employs two techniques: the nearest neighbor heuristic that is based on the $\alpha$-measure (Helsgaun 2000) and the transition function, which has already been used in ant colony algorithms (Stützle and Hoos 2000). The latter makes use of this feature: Each vertex in a Hamiltonian cycle is a vertex with degree two. After the filtering stage, a list of missing vertices and their degrees is made. Then, by manipulating this list, the algorithm connects vertices by 
using the transition function. This modification intensifies the exploration of the search space, which translates the quality of solutions into the amount of pheromone and therefore increases the proposed algorithm's adaptability. Both methods of completing the set of edges are used according to the principle: For every 50 completion operations that have been conducted by using the transition function, one iteration of the nearest neighbor heuristic is carried out. After creating a complete Hamiltonian cycle, all values representing the probability of selecting edge $a$ are reset to an initial value of one.

The dominant operation in the DPSO algorithm is the intersection of a pair of solutions, e.g., the current position of a particle and gBest (Eq. 3). The time complexity of this operation is critical for the performance of the DPSO algorithm. We adopted the following encoding. The Hamiltonian cycle is stored in an array of natural numbers in which the value at index $i$ denotes the end node of the edge starting at node $i$. This encoding allows to calculate the intersection of a pair of Hamiltonian cycles in $O(n)$ time, where $n$ denotes the size of the problem (the number of nodes). The set of velocities and partial solutions is stored as lists of 3-tuples $(a, x, y)$, where $a \in[0,1]$ denotes the probability of selecting the edge $(x, y), x, y \in V, \forall_{x, y} x \neq y$.

\section{Results}

This section consists of two parts. The first part describes the process of creating a library DTSP instances (tests). The second part presents the results of the experiments conducted. Each calculation was repeated 30 times.

\subsection{Parameters of the algorithms}

In order to objectively compare the algorithms, a constant fixed number of generated (and evaluated) solutions was adapted as the stopping criterion. On this basis, the values of the remaining parameters of the algorithms were determined, for example, the population size and the number of iterations of an algorithm. Table 1 contains list of the parameters of the investigated algorithms.

The number of solution evaluations, $p_{e v}$, for each DTSP instance equals:

$p_{e v}=p_{m u} \cdot n \cdot p_{i t} \cdot p_{p s}$

where $p_{i t}$ and $p_{p s}$ are the number of iterations of the algorithm and the swarm size, respectively, and $p_{m u}$ is a preset multiplier. The computations were carried out for a few increasing values of $p_{m u}$ and, hence, for a few different numbers of allowed solution evaluations. The total number of executed iterations $\left(p_{t e v}\right)$ was $11 \cdot p_{e v}$ because each of the
Table 1 Parameters of the algorithms

\begin{tabular}{ll}
\hline Name & Description \\
\hline$p_{i t}$ & Number of algorithm iterations \\
$p_{\text {pop }}$ & Size of the population \\
$p_{\text {sub }}$ & Number of DTSP subproblems \\
$p_{\text {ev }}$ & Number of solution evaluations \\
$p_{t e v}$ & Total number of sol. evaluations \\
$p_{m u}$ & Solution evaluations multiplier \\
$n$ & Size of the problem \\
\hline
\end{tabular}

DTSP instances consisted of 11 subproblems ( $p_{s u b}$, constant number in article). The specific values of these parameters that were used in the experiments are shown in Table 2.

The parameter values of the MMAS and PACO algorithms were chosen based on suggestions in the literature Stützle and Hoos (2000); Oliveira et al. (2011) and preliminary computations. The parameter values for the MMAS were as follows: number of ants $-m=n$, where $n$ is the size of the problem, $\beta=3, q_{0}=0.0$, size of the candidate set $c l=30$ and $\rho=0.9-$ pheromone update coefficient. For the PACO, a fixed number of ants, i.e., $p_{\text {pop }}=10$, was adapted, as a result of which the number of iterations for each subproblem of the DTSP equaled $\left\lfloor 0.1 \cdot p_{e v}\right\rfloor$. This is consistent with the observations that were presented in Cáceres et al. (2014), in which the ACO algorithms were tested for a small computational budget. The values of the other parameters for the PACO were: $\beta=3, q_{0}=0.8, c l=30, \alpha=0.1$ and $\psi=0.1-$ pheromone evaporation coefficients for local and global pheromone trail updates, respectively. Also the age-based strategy for updating the pheromone trail from an archive of solutions of size 5 was used.

As for the DPSO algorithms, the following parameters were determined (based on Table 2): the number of iterations $\left(p_{i t}\right)$ and the population size $\left(p_{p o p}\right)$, which are presented in Table 3. Table 4 shows the values of the remaining DPSO parameters.

\subsection{DPSO with and without pheromone}

During the first phase of the experiments, the DPSO algorithms with and without pheromone memory were compared using the static TSP. The aim was to evaluate how the pheromone memory affects the performance of the DPSO. If the implementation of the DPSO algorithm with pheromone allows one to obtain better results than the version without pheromone in the case of the TSP, then this implementation may also allow one to achieve better results for the DTSP. This idea can be justified by the fact that the DTSP can be seen as a sequence of static TSP instances and the information gathered about one instance in the pheromone 
Table 2 Number of solution evaluations for a single DTSP subproblem for the DPSO algorithm
Table 3 Parameters of the DPSO algorithm for all problem instances

\begin{tabular}{llllllll}
\hline Problem & & \multicolumn{6}{l}{ Evaluations $\left(p_{e v}\right)$} \\
\cline { 3 - 8 } Name & $n$ & $p_{m u}=32$ & $p_{m u}=64$ & $p_{m u}=128$ & $p_{m u}=256$ & $p_{m u}=512$ & $p_{m u}=1024$ \\
\hline berlin52 & 52 & 1664 & 3328 & 6656 & 13,312 & 26,624 & 53,248 \\
kroA100 & 100 & 3200 & 6400 & 12,800 & 25,600 & 51,200 & 10,2400 \\
kroA200 & 200 & 6400 & 12,800 & 25,600 & 51,200 & 102,400 & 204,800 \\
gr202 & 202 & 6464 & 12,928 & 25,856 & 51,712 & 103,424 & 206,848 \\
gr666 & 666 & 21,312 & 42,624 & 85,248 & 170,496 & $1,875,456$ & 681,984 \\
\hline
\end{tabular}

The values were calculated according to the formula: $p_{m u} \cdot n$

\begin{tabular}{|c|c|c|c|c|c|c|c|c|c|c|c|c|}
\hline \multirow[t]{2}{*}{ Problem } & \multicolumn{2}{|c|}{$p_{m u}=32$} & \multicolumn{2}{|c|}{$p_{m u}=64$} & \multicolumn{2}{|c|}{$p_{m u}=128$} & \multicolumn{2}{|c|}{$p_{m u}=256$} & \multicolumn{2}{|c|}{$p_{m u}=512$} & \multicolumn{2}{|c|}{$p_{m u}=1024$} \\
\hline & $p_{i t}$ & $p_{\text {pop }}$ & $p_{i t}$ & $p_{\text {pop }}$ & $p_{i t}$ & $p_{\text {pop }}$ & $p_{i t}$ & $p_{\text {pop }}$ & $p_{i t}$ & $p_{\text {pop }}$ & $p_{i t}$ & $p_{p o p}$ \\
\hline berlin52 & 52 & 32 & 104 & 32 & 208 & 32 & 416 & 32 & 832 & 32 & 1664 & 32 \\
\hline kroA 100 & 50 & 64 & 100 & 64 & 200 & 64 & 400 & 64 & 800 & 64 & 1600 & 64 \\
\hline kroA200 & 80 & 80 & 160 & 80 & 320 & 80 & 640 & 80 & 1280 & 80 & 2560 & 80 \\
\hline gr202 & 64 & 101 & 128 & 101 & 256 & 101 & 512 & 101 & 1024 & 101 & 2048 & 101 \\
\hline gr666 & 192 & 111 & 384 & 111 & 768 & 111 & 1536 & 111 & 3072 & 111 & 6144 & 111 \\
\hline
\end{tabular}

Table 4 Parameters of the DPSO algorithm with and without pheromone

\begin{tabular}{|c|c|c|c|c|c|c|c|c|c|c|c|c|}
\hline \multirow[t]{3}{*}{ Problem } & \multicolumn{7}{|c|}{ Zhong et al. (1997) DPSO } & & \multirow{2}{*}{\multicolumn{4}{|c|}{$\begin{array}{l}\text { DPSO with pheromone } \\
\text { Paper settings }\end{array}$}} \\
\hline & \multicolumn{4}{|c|}{ Paper settings } & \multicolumn{4}{|c|}{ Original setting } & & & & \\
\hline & $c_{1}$ & $c_{2}$ & $c_{3}$ & $\omega$ & $c_{1}$ & $c_{2}$ & $c_{3}$ & $\omega$ & $c_{1}$ & $c_{2}$ & $c_{3}$ & $\omega$ \\
\hline berlin 52 & 1.5 & 2 & 2 & 0.6 & 0.5 & 0.5 & 0.5 & 0.2 & 0.5 & 0.5 & 0.5 & 0.2 \\
\hline kroA100 & 1.5 & 2 & 2 & 0.6 & 0.5 & 0.5 & 0.5 & 0.5 & 0.5 & 0.5 & 0.5 & 0.5 \\
\hline kroA200 & 1.5 & 2 & 2 & 0.6 & 0.5 & 0.5 & 0.5 & 0.5 & 0.5 & 0.5 & 0.5 & 0.5 \\
\hline gr202 & 1.5 & 2 & 2 & 0.6 & 0.5 & 0.5 & 0.5 & 0.5 & 0.5 & 0.5 & 0.5 & 0.5 \\
\hline gr666 & 1.5 & 2 & 2 & 0.6 & 0.5 & 1 & 1.5 & 0.6 & 0.5 & 1 & 1.5 & 0.6 \\
\hline
\end{tabular}

memory may be useful in the context of a modified (next) instance.

Figure 4 presents the convergence of the two versions of the DPSO algorithm (with and without pheromone) to the optimum for five selected instances of the TSP. In the context of a given instance, calculations were repeated with an increasing number of iterations (in accordance with Table 3); they were repeated 30 times.

The sets of parameter values are marked with Roman numerals. The numeral "I" denotes values that were adapted for the calculations carried out for the purpose of this paper. These values were determined based on the results of preliminary experiments. The values of the parameters of the DPSO (marked as II) that were proposed in Zhong et al. (1997) are also presented here for comparison. In the version without pheromone, the settings that were proposed in Zhong et al. (1997) (Zhg(II)) allow one to obtain better results than the settings for the version with pheromone (Zhg(I)). This is because the algorithm with pheromone complements the probability of choosing a given edge with pheromone reinforcement. The values of scaling parame- ters $c_{1}, c_{2}, c_{3}, \omega$ should be lower, unlike in the algorithm version without pheromone, where this reinforcement does not occur. Nonetheless, this comparison was necessary as these settings were contrasted with those that were used in the version with pheromone. The two versions of this algorithm have different convergence characteristics. The version with pheromone returns better results for a larger number of iterations, which is due to the pheromone matrix's demand for learning. This is disadvantageous when the size of the search space and the number of iterations are small. As for the gr666 problem, i.e., when the search space is larger, the algorithm without pheromone allowed one to obtain better results only for the smallest number of iterations (192). In any other case, the algorithm version with pheromone produced better results. It was this algorithm that found the best solution for each problem (without taking the number of iterations into account). The influence of the growing number of iterations on the quality of the obtained solutions (the distance from the optimum) is also important. This is particularly visible for larger search spaces (from the kroA100 problem). As for the implementation of the DPSO algorithm 


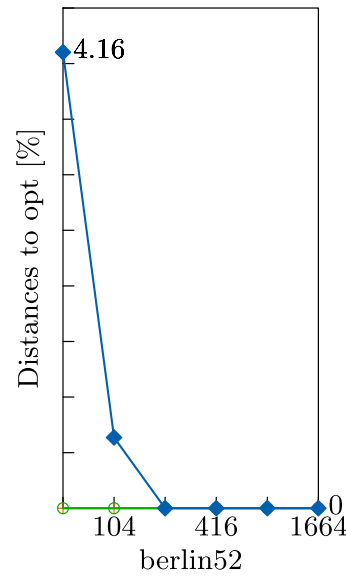

(a)

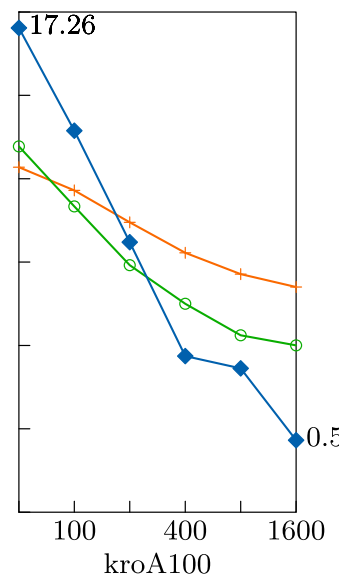

(b)

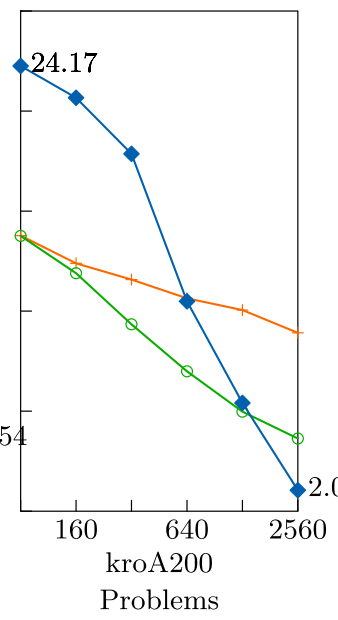

(c)

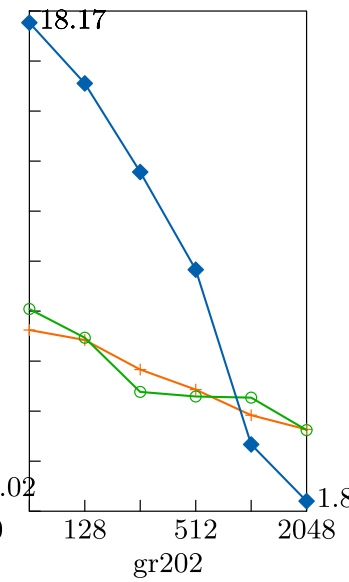

(d)

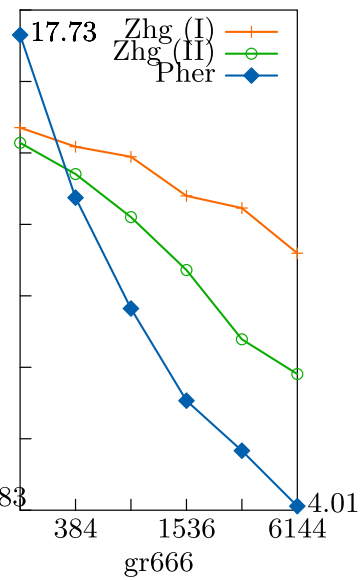

(e)

Fig. 4 Convergence of the DPSO algorithms to the optimum relative to the number of iterations. "Pher" and "Zhg" denote the implementation of the algorithm with and without pheromone, respectively. Roman numerals (I and II) refer to the values in Table 4

with pheromone, there is a large improvement (the line of convergence is almost vertical). The improvement is not visible for the implementation of the DPSO algorithm without pheromone.

\subsection{Comparison of the variants of the DPSO}

The next series of experiments entailed a comparison of two variants of the DPSO algorithm with pheromone in terms of convergence: one that involves resetting the pheromone matrix (DPSOR+) after each change of the input data and the other one that does not involve resetting the matrix (DPSOR-), in the context of the kroA200 instance from the DTSP repository for two different values of changes in the coordinates of vertices ( 3 and $50 \%$ ). In the former case, the DPSOR+ algorithm produced results that were $1.95 \%$ better (Fig. 5) than those obtained by the version that involves resetting the pheromone matrix (kroA200,3\% of changes in each subproblem). In the latter case (Fig. 5), the difference was $-0.23 \%$, in favor of the variant that involves resetting the pheromone value after each change of the input data (kroA200, 50\% of changes in each subproblem). In the last iteration of the algorithm (before the change of the data), the best solution is marked and information is provided on the variant of the algorithm's implementation that produced that solution. The two charts show different characteristics of convergence to the optimum, except for the first subproblem, for which the pheromone matrix in both these algorithms was initialized with the same (initial) values.

It can be seen from Fig. 5 that the variant that does not involve resetting the matrix in the first iterations of the algo- rithm has better convergence and that it found a solution that was not much different from the final result. Since the new optimum differs from the previous one in terms of only $10 \%$ of edges, the "knowledge about the problem" that has been gathered in the form of pheromone is mostly up to date and it improves the convergence of the algorithm, especially at the initial stage. In subsequent iterations, the pheromone matrix slowly adapts to the new data. After half of all iterations are executed, the convergence rate increases again. As convergence is fast at the beginning, the algorithm has more time to find edges that are elements of the optimal solution. This is why the $\mathrm{R}-$ version (which did not involve resetting the pheromone value) achieved better results for 9 out of 11 subproblems.

As shown in Fig. 6, pheromone did not accumulate on good quality edges due to a large number of changes $(50 \%)$ (there was a large number of changes in the optimum relative to the previous optimum, i.e., from before the changes took place). Therefore, both variants of the algorithm had to adapt the pheromone for new data, and therefore, they explored the best edges that had been found previously to a lesser extent. This does not have an impact on how many better solutions are found (a total of 8 per 11 subproblems), but it does influence a difference that is expressed in percentage points, which is negative and whose absolute value is small (0.23). Therefore, the profit from copying the pheromone matrix (transferring knowledge about the previous solution) was negative. Given that the value of the mean difference was negative and the absolute value was small, it can be stated that the benefit of retaining previous pheromone values was negligible in this case. 


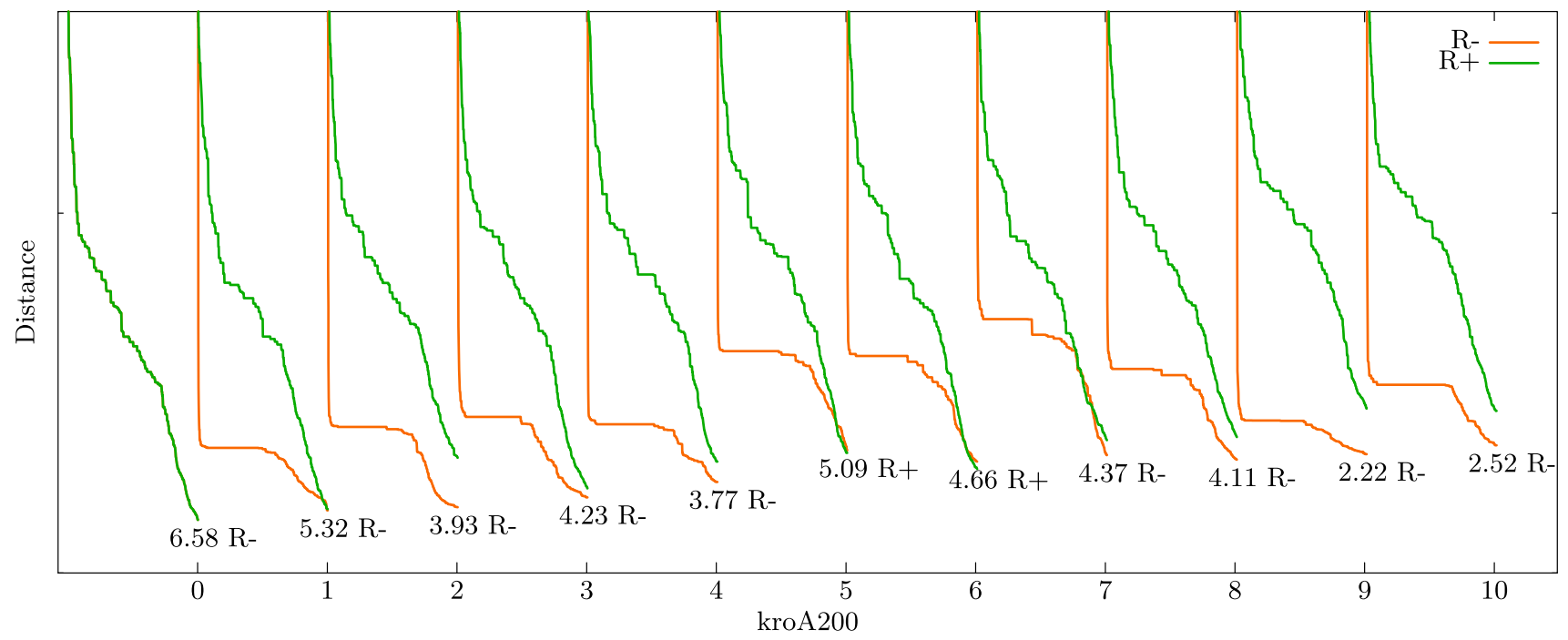

Subproblem number

Fig. 5 Convergence of the DPSO algorithms to the optimum for the problem kroA200 with $3 \%$ of changes. $\mathrm{R}+$ and $\mathrm{R}-$ denote the version that involves resetting the pheromone value and the version that does not, respectively. $X$-axis tick marks denote the moments at which the location of some of the coordinates of vertices (cities) changes

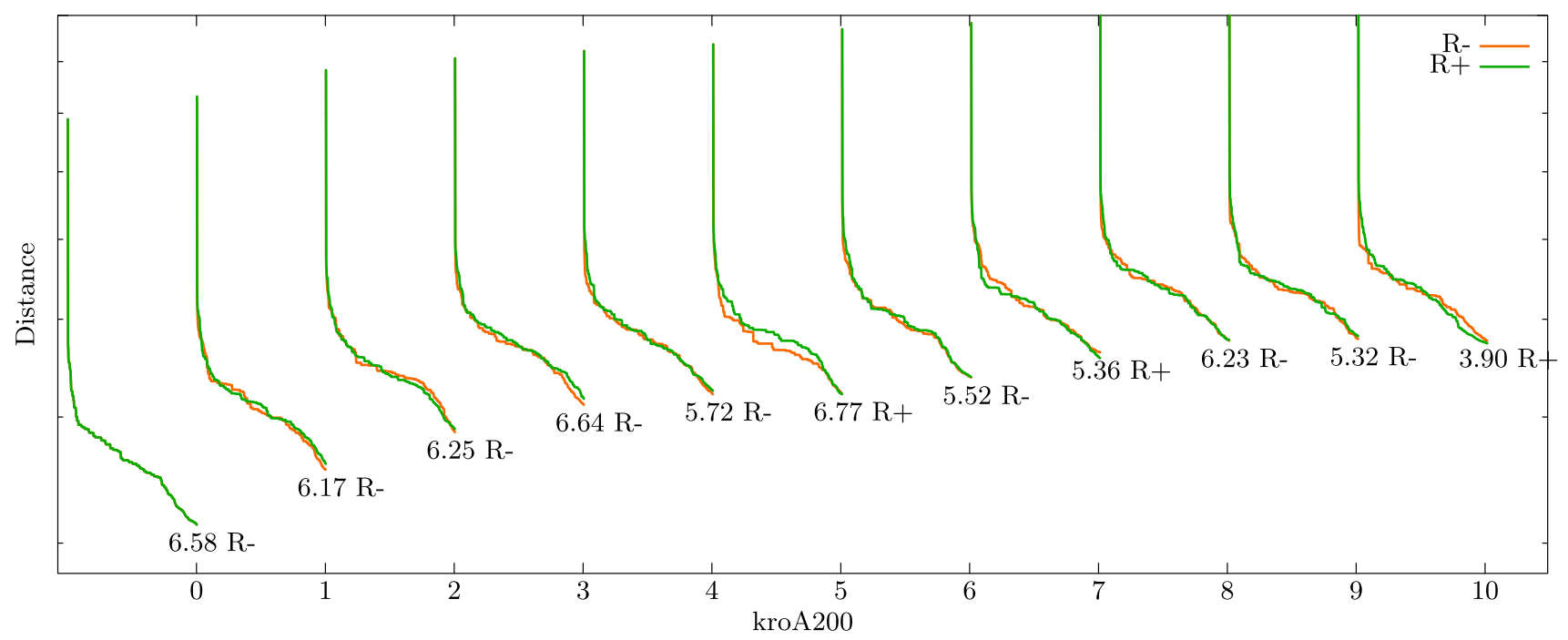

Subproblem number

Fig. 6 Convergence of the DPSO algorithm to the optimum for the problem $k r o A 200$ with $50 \%$ of changes. R+ and R- denote the version that involves resetting the pheromone value and the version that does not, respectively

\subsubsection{Influence of the number of iterations on the convergence of algorithms}

The quality of the solutions that are generated by heuristic algorithms significantly depends on the number of generated solutions. In order to test the convergence of the analyzed variants of the DPSO algorithm, i.e., those that involve resetting pheromone memory and those that do not, a range of calculations were carried out for different numbers of iterations of the algorithm which had been determined in accordance with Table 3. Figures 7 and 8 present charts showing average quality of the solutions that were obtained for versions of the DPSO algorithm that did and did not involve resetting pheromone memory, respectively. As can be seen, together with an increase in the number of iterations of the algorithm, the quality of solutions that are generated improves significantly, and the largest relative improvement in convergence can be observed for smaller values of the iteration multiplier $\left(p_{m u}\right)$. If the number of iterations was further increased, this would certainly improve the quality of solu- 

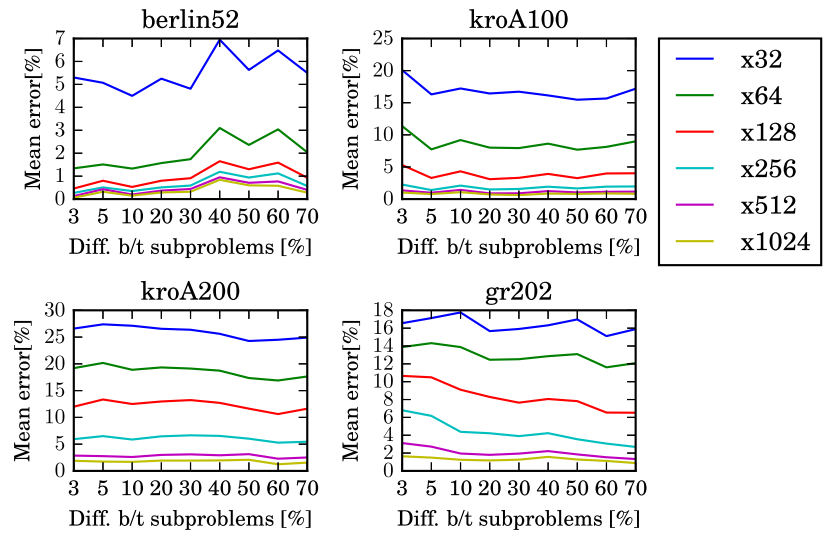

Fig. 7 A comparison of mean relative errors of solutions for the tests berlin52, kroA100, kroA200 and gr202 in terms of the percentage of the number of vertices that underwent changes for the DPSOR+ algorithm (i.e., the DPSO algorithm that involves resetting the pheromone value). The consecutive series of data on the charts denote the results for different values of multipliers, in accordance with Table 3
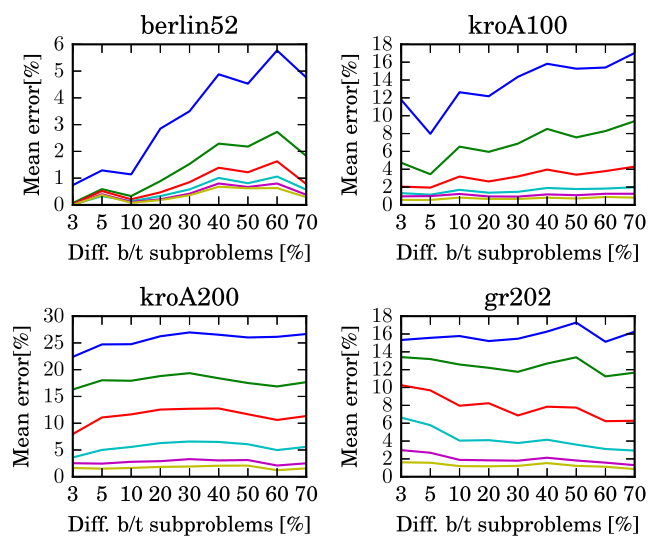

Diff. b/t subproblems [\%]

Fig. 8 A comparison of mean relative errors of solutions for the tests berlin52, kroA100, kroA200 and gr202 in terms of the percentage of the number of vertices that underwent changes for the DPSOR - algorithm (i.e., the DPSO algorithm that does not involve resetting the pheromone value)

tions, but the change would be relatively small, which one can observe by comparing the results for the values of the multiplier: 512 and 1024.

The dominance of the DPSOR - algorithm, in which pheromone memory is not reset after changes that are made to the current subproblem, is also evident. This is particularly visible for the smallest problems, i.e., berlin52 and kroA100.

\subsection{Comparison of all algorithms}

In order to compare the DPSO algorithms with the MMAS and the PACO, a range of computational experiments was conducted for a set of four instances: berlin52, kroA100, kroA200 and gr202. To check the "extent of usefulness" of pheromone memory for the search process, the behavior of algorithms was tested for the instances of the problem with an increasing percentage $(3,5,10,20,30,40,50,60$ and $70 \%$ ) of the total number of vertices (cities) whose coordinates underwent random changes. Intuitively, when only a small proportion of vertices change their location, the existing knowledge about the search space that is stored in pheromone trails largely remains up to date and makes it easier to find high-quality solutions for a new subproblem. If, however, the extent of changes is very large, for example $60 \%$, one can expect that "old" knowledge about the problem will mostly be outdated.

Figure 9 presents a box plot of the mean solution error for the DPSO algorithms that involve resetting the pheromone matrix (DPSOR+) and the DPSO algorithms that do not involve resetting the pheromone matrix as well as for the MMAS and PACO algorithms for the problem berlin52. As can be seen, the DPSO algorithm outperforms the other algorithms, especially when the number of vertices whose coordinates undergo modifications between consecutive subproblems is small. The version of the DPSO algorithm that does not involve resetting the pheromone matrix turned out to be much better than the DPSO algorithm version that does

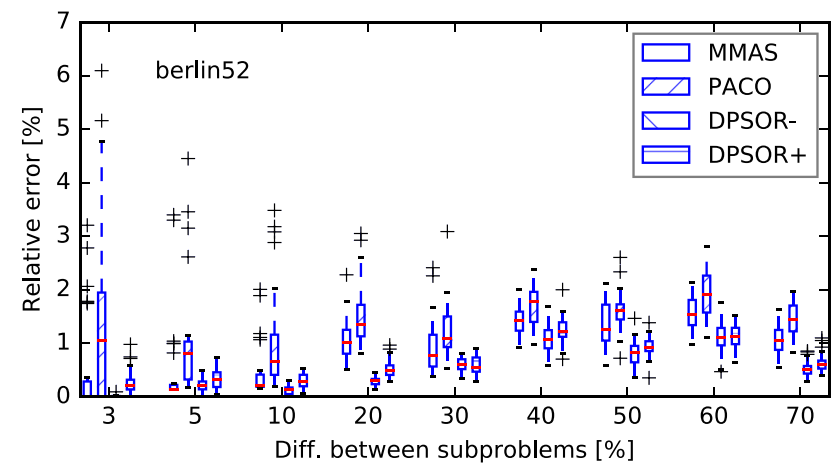

Fig. 9 Box plot of the relative solution error for the berlin52 DTSP instance for the analyzed algorithms. The boxes are grouped according to the percent of the total number of vertices (cities) whose locations underwent a change

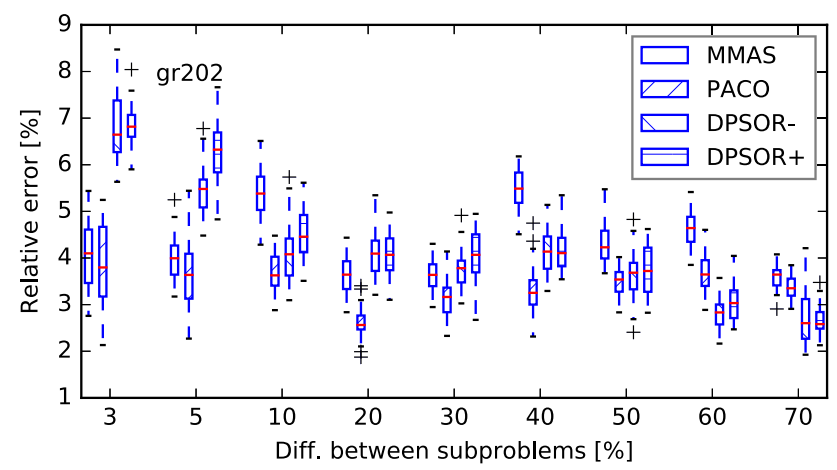

Fig. 10 Box plot of the relative solution error for the kroA200 DTSP instance for the analyzed algorithms. The boxes are grouped according to the percent of the total number of vertices (cities) whose locations underwent a change 


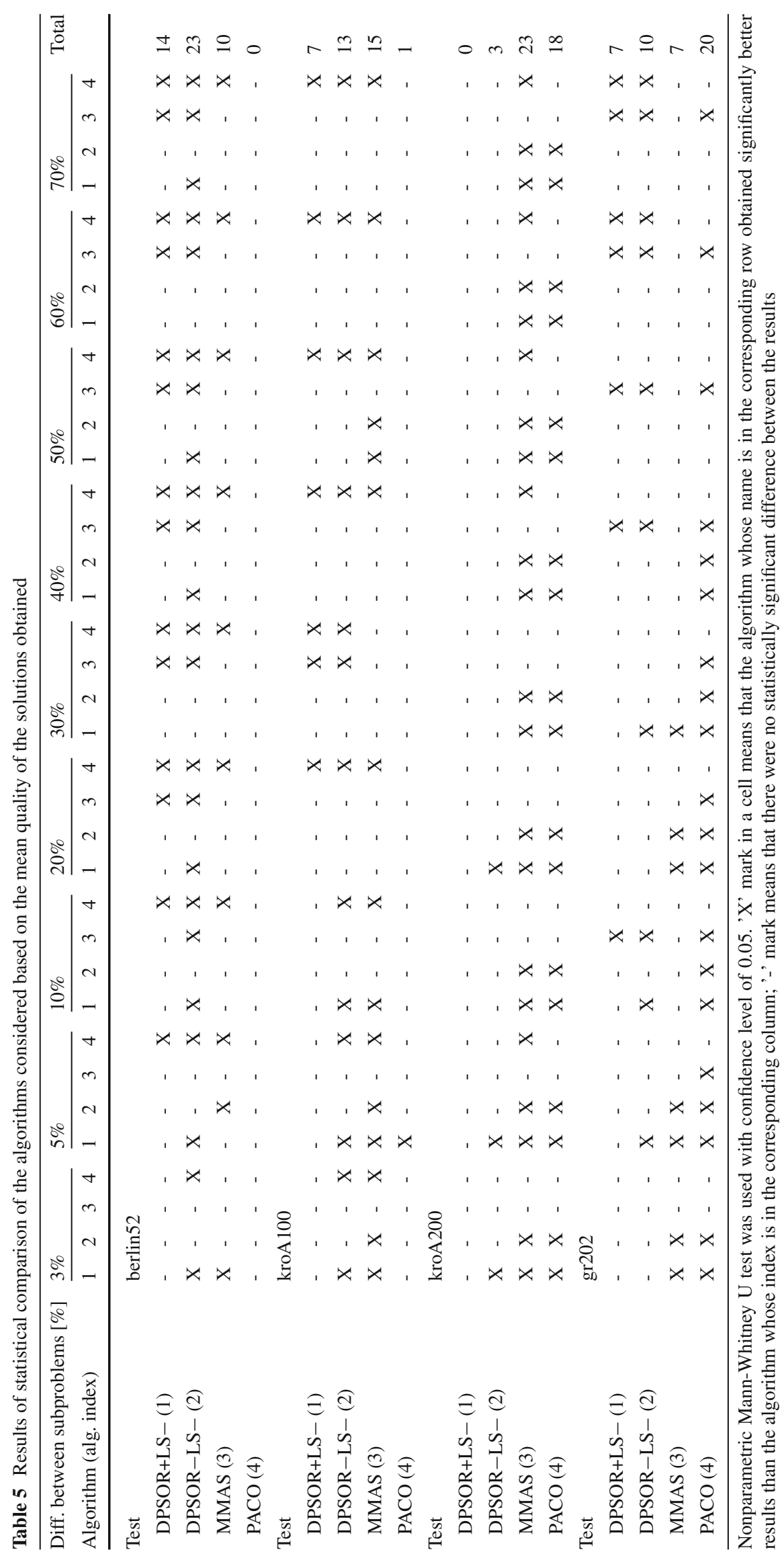


involve resetting the pheromone value when the percentage of the number of vertices that underwent changes was not higher than $20 \%$.

A comparison of the algorithms for the $g r 202$ instance, which is presented in Fig. 10, is more interesting. ACO algorithms achieved better results when the problem underwent small changes, i.e., changes that did not amount to more than $5 \%$, whereas the DPSO algorithm proved to be better when the changes were significant $(60$ and $70 \%)$. This is because the adapted parameter values of the MMAS and PACO algorithms resulted in putting a large emphasis on the exploitation of the search space around the best solutions that had been obtained at the expense of more extensive exploration.

All the algorithms were compared in terms of the quality of the results that were produced based on a two-sided, nonparametric Mann-Whitney-Wilcoxon test, with a significance level of $5 \%$. The results of the calculations for the number of evaluations $p_{e v}=256 \cdot 11 \cdot n$ are summarized in Table 5 . These results vary significantly for some instances. The MMAS algorithm performed significantly better more often than the other algorithms for the kroA 100 and kroA200 instances. The DPSO algorithm, which did not involve resetting the pheromone matrix, obtained good results for the berlin52 and kroA100 instances. The performance of the PACO was especially good for the gr202 instance. When taking into account all four instances, the MMAS obtained significantly better results in 55 cases, the DPSOR- algorithm (i.e., the DPSO version of algorithm, which did not involve resetting the pheromone value) in 49 cases, the PACO algorithm in 39 cases and the DPSOR+ algorithm in 28 cases.

To recapitulate, the ant colony algorithms turned out to be better in a larger number of cases, even though the parameter values of the DPSO were chosen on a per-instance basis, as indicated in Table 4. Nevertheless, the performance of the DPSO algorithms, particularly the DPSOR-, is encouraging, especially considering the fact that the DTSP can be considered a native as problem to the ACO algorithms, i.e., it is discrete and graph based.

\section{Conclusions}

Dynamic optimization problems have great practical significance. An innovative algorithm for discrete particle swarm optimization (DPSO) is proposed in the present paper; this algorithm has been enriched by pheromone memory which is modeled on ant algorithms. The DPSO searches the solution space because of pheromone that makes use of machine learning and due to the interaction between particles. In this way, it combines the advantages of ant colony algorithms and classical particle swarm optimization. For the purpose of computational experiments, a library of DTSP instances was developed based on the well-known TSPLIB library (Reinelt 1995). For each test, a dynamic counterpart was prepared which consisted of a series of subproblems that had been created as a result of a random change in the location of a predetermined number of coordinates of cities (vertices). For each subproblem, an optimal solution was determined, which allowed one to clearly evaluate the quality of the results that were obtained for the analyzed algorithms.

The algorithms were tested on four different DTSP instances with 9 different intensities of changes between consecutive subproblems as well as for 6 different limits on the number of generated solutions (in total, there were $216 \mathrm{com}$ binations). The quality of the results greatly depended on the computational budget that had been adapted. The average quality of solutions was within $1 \%$ from optima for the larger numbers of solutions created. The quality of solutions could have been significantly improved if local search had been applied.

It is worth noting that the use of pheromone memory improves the convergence of the DPSO algorithm for the DTSP. If the differences (coordinates of points) between consecutive subproblems of the DTSP are relatively small, then the knowledge about the previous subproblem that is accumulated in pheromone memory makes it easier to find good solutions for a new subproblem. This is particularly visible when the computational budget that has been adapted is small, which confirms that this algorithm is useful when the problem undergoes frequent changes and the time periods between consecutive changes does not make it possible to carry out long calculations. On the other hand, if the problem rarely undergoes modifications, similar quality results can be obtained by using the DPSO algorithm, in which pheromone memory is reset following each modification of the problem and the algorithm execution is equivalent to separate executions of this algorithm for each of the DTSP's subproblems.

Although the MMAS and PACO algorithms produced better results in a larger number of cases, this advantage is not big, which shows that the DPSO algorithm is competitive. Further studies should take into account local search heuristics and focus on solving larger DTSP instances (with thousands of cities). It will also be interesting to use the DPSO algorithm for other dynamic combinatorial optimization problems, such as the dynamic vehicle routing problem.

Acknowledgements This research was supported in part by PL-Grid Infrastructure.

\section{Compliance with ethical standards}

Conflict of interest The authors declare that they have no potential conflict of interest.

Open Access This article is distributed under the terms of the Creative Commons Attribution 4.0 International License (http://creativecomm ons.org/licenses/by/4.0/), which permits unrestricted use, distribution, 
and reproduction in any medium, provided you give appropriate credit to the original author(s) and the source, provide a link to the Creative Commons license, and indicate if changes were made.

\section{References}

Applegate D, Bixby R, Chvatal V, Cook W (2006) Concorde TSP solver. http://www.math.uwaterloo.ca/tsp/concorde.html. Accessed 24 Jul 2017

Bilu Y, Linial N (2012) Are stable instances easy? Comb Probab Comput 21(5):643-660

Blackwell T, Branke J, Li X (2008) Particle swarms for dynamic optimization problems. In: Blum C, Merkle D (eds) Swarm Intelligence. Natural computing series. Springer, Berlin, Heidelberg, pp 193-217

Boryczka U, Strạk Ł (2012) A hybrid discrete particle swarm optimization with pheromone for dynamic traveling salesman problem. In: Computational collective intelligence. Technologies and applications, lecture notes in computer science, vol 7654. Springer, Berlin, Heidelberg, pp 503-512

Boryczka U, Strąk Ł (2013) Efficient DPSO neighbourhood for dynamic traveling salesman problem. In: Computational collective intelligence. Proceedings on Technologies and applications-5th international conference, ICCCI 2013, Craiova, Romania, September 11-13, pp 721-730

Boryczka U, Strạk Ł (2015a) Diversification and entropy improvement on the dpso algorithm for dtsp. In: Intelligent information and database systems, lecture notes in computer science, vol 9011. Springer International Publishing, Berlin, pp 337-347

Boryczka U, Strąk $€$ (2015b) Heterogeneous dpso algorithm for dtsp. In: Computational collective intelligence, lecture notes in computer science, vol 9330. Springer International Publishing, pp 119-128

Cáceres LP, López-Ibánez M, Stützle T (2014) Ant colony optimization on a budget of 1000. In: Swarm intelligence, Springer, pp 50-61

Demirtaş YE, Özdemir E, Demirtaş U (2015) A particle swarm optimization for the dynamic vehicle routing problem. In: 2015 6th International conference on modeling, simulation, and applied optimization (ICMSAO). IEEE, pp 1-5

Dorigo M, Stützle T (2010) Ant colony optimization: overview and recent advances. In: Gendreau M, Potvin JY (eds) Handbook of metaheuristics. Springer, pp 227-263

Eyckelhof CJ, Snoek M, Vof M (2002) Ant systems for a dynamic tsp: ants caught in a traffic jam. In: Ant algorithms: third international workshop, ANTS 2002, vol 2463/2002 of lecture notes in computer science. Springer, pp 88-99

Goldbarg E, de Souza G, Goldbarg M (2008) Particle swarm optimization algorithm for the traveling salesman problem. INTECH Open Access Publisher, Rijeka

Guntsch M, Middendorf M (2001) Pheromone modification strategies for ant algorithms applied to dynamic tsp. In: Boers EJW (ed) Applications of evolutionary computing. Springer, pp 213-222

Guntsch M, Middendorf M (2002) Applying population based aco to dynamic optimization problems. In: Ant Algorithms, Springer, pp $111-122$

Guntsch M, Middendorf M, Schmeck H (2001) An ant colony optimization approach to dynamic tsp. In: Proceedings of the 3rd annual conference on genetic and evolutionary computation. Morgan Kaufmann Publishers Inc., San Francisco, CA, USA, GECCO'01, pp 860-867. http://dl.acm.org/citation.cfm?id=2955239.2955396

Helsgaun K (2000) An effective implementation of the Lin-Kernighan traveling salesman heuristic. Eur J Oper Res 126:106-130

Hu X, Shi Y, Russell E (2004) Recent advances in particle swarm. In: Congress on evolutionary computation, CEC2004, vol 1, pp 90-97
Kalivarapu V, Foo JL, Winer E (2009) Improving solution characteristics of particle swarm optimization using digital pheromones. Struct Multidiscip Optim 37(4):415-427

Kang L, Zhou A, McKay RI, Li Y, Kang Z (2004) Benchmarking algorithms for dynamic travelling salesman problems. In: Proceedings of the IEEE congress on evolutionary computation, CEC 2004, 19-23 June 2004, Portland, OR, USA, pp 1286-1292

Kennedy J, Eberhart R (1995) Particle swarm optimization. In: Proceedings of the IEEE international conference on neural networks, pp $1942-1948$

Khouadjia MR, Jourdan L, Talbi EG (2010) Adaptive particle swarm for solving the dynamic vehicle routing problem. In: 2010 IEEE/ACS international conference on computer systems and applications (AICCSA). IEEE, pp 1-8

Li W (2011) A parallel multi-start search algorithm for dynamic traveling salesman problem. In: Proceedings of the 10th international conference on experimental algorithms

Li C, Yang M, Kang L (2006) A new approach to solving dynamic traveling salesman problems. In: Proceedings of the 6th international conference on simulated evolution and learning. Springer, Berlin, Heidelberg, SEAL'06, pp 236-243

Mavrovouniotis M, Yang S (2010) Ant colony optimization with immigrants schemes in dynamic environments. In: Schaefer R, Cotta C, Kołodziej J, Rudolph G (eds) Parallel problem solving from nature, PPSN XI, lecture notes in computer science, vol 6239. Springer, Berlin, Heidelberg, pp 371-380

Mavrovouniotis M, Yang S, Yao X (2012) A benchmark generator for dynamic permutation-encoded problems. Springer, Berlin

Mavrovouniotis M, Li C, Yang S (2017) A survey of swarm intelligence for dynamic optimization: algorithms and applications. Swarm Evolut Comput 33:1-17

Mori N, Kita H (2000) Genetic algorithms for adaptation to dynamic environments - a survey. In: Industrial electronics society, 2000. IECON 2000, 26th annual conference of the IEEE, vol 4, pp $2947-$ 2952

Okulewicz M, Mańdziuk J (2013) Application of particle swarm optimization algorithm to dynamic vehicle routing problem. In: International conference on artificial intelligence and soft computing. Springer, pp 547-558

Oliveira SM, Hussin MS, Stützle T, Roli A, Dorigo M (2011) A detailed analysis of the population-based ant colony optimization algorithm for the tsp and the qap. In: Proceedings of the 13th annual conference companion on Genetic and evolutionary computation. ACM, pp 13-14

Pedemonte M, Nesmachnow S, Cancela H (2011) A survey on parallel ant colony optimization. Appl Soft Comput 11(8):5181-5197. doi:10.1016/j.asoc.2011.05.042

Pintea CM, Pop PC, Dumitrescu D (2007) An ant-based technique for the dynamic generalized traveling salesman problem. In: Proceedings of the 7-th WSEAS international conference on systems theory and scientific computation, pp 257-261

Pintea C, Crisan GC, Manea M (2012) Parallel ACO with a ring neighborhood for dynamic TSP. JITR 5(4):1-13. doi:10.4018/jitr. 2012100101

Pop PC, Pintea C, Dumitrescu D (2009) An ant colony algorithm for solving the dynamic generalized vehicle routing problem. Civil Eng 1(11):373-382

Psaraftis H (1988) Dynamic vehicle routing problems. Veh Routing Methods Stud 16:223-248

Reinelt G (1995) TSPLIB95. Interdisziplinäres Zentrum für Wissenschaftliches Rechnen (IWR). Heidelberg

Stützle T, Hoos HH (2000) Max-min ant system. Future Gener Comput Syst 16(8):889-914

Tinós R, Whitley D, Howe A (2014) Use of explicit memory in the dynamic traveling salesman problem. In: Proceedings of the 2014 annual conference on genetic and evolutionary computation. 
ACM, New York, NY, USA, GECCO '14, pp 999-1006. doi:10. $1145 / 2576768.2598247$

Yang S, Yao X (2013) Evolutionary computation for dynamic optimization problems. Springer, Berlin

Younes A, Basir O, Calamai P (2003) A benchmark generator for dynamic optimization. In: Digest of the Proceedings of the wseas conferences

Younes A, Calamai P, Basir O (2005) Generalized benchmark generation for dynamic combinatorial problems. In: Proceedings of the 7th annual workshop on genetic and evolutionary computation ACM, New York, NY, USA, GECCO '05, pp 25-31. doi:10.1145/ 1102256.1102262
Zhong Wl, Zhang J, Chen Wn (1997) A novel set-based particle swarm optimization method for discrete optimization problems. In: Evolutionary computation, 2007. CEC 2007, vol 14. IEEE, pp $3283-3287$ 